Christian Neuhuber, Stefanie Endler, Elisabeth Zehetner, Bairisch-österreichische Dialektliteratur vor 1800. Eine andere Literaturgeschichte

\title{
Wolfgang Pöckl
}

\section{(2) OpenEdition}

Journals

Édition électronique

URL : https://journals.openedition.org/austriaca/910

DOI : 10.4000/austriaca.910

ISSN : 2729-0603

Éditeur

Presses universitaires de Rouen et du Havre

\section{Édition imprimée}

Date de publication : 1 décembre 2019

Pagination : $275-279$

ISBN : 979-10-240-1454-8

ISSN : 0396-4590

Référence électronique

Wolfgang Pöckl, „Christian Neuhuber, Stefanie Endler, Elisabeth Zehetner, Bairisch-österreichische Dialektliteratur vor 1800. Eine andere Literaturgeschichte“, Austriaca [Online], 88-89 | 2019, Online erschienen am: 01 Juli 2020, abgerufen am 24 Oktober 2022. URL: http://journals.openedition.org/ austriaca/910 ; DOI: https://doi.org/10.4000/austriaca.910

Ce document a été généré automatiquement le 24 octobre 2022.

All rights reserved 


\title{
Christian Neuhuber, Stefanie Endler, Elisabeth Zehetner, Bairisch- österreichische Dialektliteratur vor 1800. Eine andere Literaturgeschichte
}

\author{
Wolfgang Pöckl
}

\section{RÉFÉRENCE}

Christian Neuhuber, Stefanie Endler, Elisabeth Zehetner, Bairisch-österreichische Dialektliteratur vor 1800. Eine andere Literaturgeschichte, Wien-Köln-Weimar, Böhlau, 2019, 649 p., ISBN : 978-3-205-20630-9.

1 Es ist ein imposantes Kompendium, das hier von einem an der Universität Graz tätigen Germanistenteam vorgelegt wird. Die im knappen Umschlagtext angesprochenen Verdienste dieser Literaturgeschichte kann man ohne Einschränkung unterschreiben: „Das Buch präsentiert erstmals die wichtigsten Ausdrucksformen, Arbeiten und Autoren, ${ }^{1}$ kontextualisiert die bislang überwiegend nicht edierten Werke aus einer Vielzahl an Archiven und Bibliotheken und stellt sie in aktuelle kulturwissenschaftliche Zusammenhänge."

2 Der von den Verfassern nicht einlässlicher thematisierte Untertitel „Eine andere Literaturgeschichte" hat in mehrfacher Hinsicht seine volle Berechtigung. Im Gegensatz zur prototypischen Vertreterin dieser Textsorte, die die Höhenkammliteratur und deren Autorinnen und Autoren privilegiert, literarische Strömungen in chronologischer Abfolge darstellt und die jeweils repräsentativen Werke nach Gattungen sortiert, ist das dokumentierte und kommentierte Material hier vorrangig nach inhaltlichen Kriterien geordnet. Innerhalb der acht Hauptkapitel 1) Glaube und Aberglaube, 2) Krieg und Frieden, 3) Herrschaft und Untertan, 4) Land- und Stadtleben, 5) Fremdes und Eigenes, 6) Brauchtum und Geselligkeit, 7) Körper und Sinnlichkeit, 
8) Fehden und Feindschaften erfolgt die funktionale Ausdifferenzierung der Textgruppen. So wird beispielsweise Kapitel 1 untergliedert in: Lieder und Spiele des Weihnachtskreises (der Titel signalisiert bereits, dass die Gattungszugehörigkeit kein entscheidendes Einteilungskriterium darstellt), Bibelhumoresken, Heiligen- und Legendenspiele, Andachtstexte und Kritisch-Komisches zu Glaubenslehren, -leuten und -dingen. Nur im zweiten Kapitel, wo die Mehrzahl der Texte zu konkreten kriegerischen Auseinandersetzungen in Beziehung gesetzt wird, drängt sich eine chronologische Abfolge auf (Großer Türkenkrieg, Spanischer Erbfolgekrieg, Österreichischer Erbfolgekrieg, Siebenjähriger Krieg etc.). Aber auch in diesem Abschnitt wird dem ereignisübergreifenden Thema der Soldatenwerbung eine separate Betrachtung gewidmet.

3 Da die Texte - bis auf wenige Ausnahmen - dem mündlichen Überlieferungsstrang angehören und oft wohl nur zufällig (in einer von mehreren in Umlauf befindlichen und der jeweiligen Gebrauchssituation angepassten Version) aufgezeichnet wurden, ${ }^{2}$ wäre die Rekonstruktion einer "Urform“ und die Spekulation über den eventuellen Verfasser (dessen Profil in der Regel auch äußerst unscharf bliebe) schwer möglich und auch unergiebig. Im umfangreichen Personenregister finden sich zwar viele Namen, da die Verfasser der Literaturgeschichte den Stand der Forschung zu einzelnen Texten zu dokumentieren bemüht sind und auch tentative Zuschreibungen meist am Rande erwähnen, es sind aber nur sehr wenige prominente und produktive Textproduzenten registriert. Eine Ausnahme bildet hier der Lambacher Benediktiner Maurus Lindemayr, dessen Biographie relativ gut bekannt ist. Mit großem Abstand bei den Nennungen folgt Joseph Richter, bei dem es sich nach den Worten der Verfasser um den „wohl meistgelesenen josephinistischen Unterhaltungsschriftsteller" (S. 216f.) handelt. Auch der österreichische Aufklärungsautor Aloys Blumauer wird in verschiedenen Zusammenhängen erwähnt. Interessanterweise trifft man im Namenindex aber auf eine Reihe höchst namhafter Komponisten - Mozart, Joseph und Michael Haydn, Johann Georg Albrechtberger -, durch deren Vertonung auch eher marginale und bisweilen gar nicht salonfähige Texte zum musikalischen Scherz und damit in gewisser Weise zum bewahrungswürdigen Kulturgut mutierten.

Das Hauptaugenmerk liegt das gesamte Werk hindurch natürlich auf der Frage, welche Funktionen der Dialekt in welchen Kontexten erfüllt. Hier zeigt sich eine große Bandbreite an Möglichkeiten. Im religiösen Bereich etwa sind die Aufgaben von Sprachen bzw. Varietäten im Allgemeinen recht eindeutig verteilt. In der Liturgie dominiert Latein als (vom einfachen Gläubigen weithin unverstandener) Kultolekt, Teile der Messe - insbesondere die Predigt - wurden im (zunächst oberdeutschen, im Lauf des 18. Jahrhunderts immer stärker mitteldeutschen) Standard zelebriert. Für den Dialekt eröffneten sich nur kleine Nischen: etwa im risus paschalis oder, in Spurenelementen, in der homiletischen Praxis. Außerhalb der liturgischen Zeremonien und des täglichen Gebets aber, wenn (vornehmlich zur Advents- und Weihnachtszeit) Szenen aus der Bibel zur didaktisch unterlegten Unterhaltung dramatisch ausgestaltet wurden, bedienten sich die Engel (sowie meist auch Maria und Josef) der Standardsprache, während das Hirtenvolk als sprachliches Identifikationsangebot an die breite Bevölkerung den vertrauten Dialekt verwendete. Diese Tradition hat sich in ländlichen Gebieten bis heute erhalten - vielleicht mit der Einschränkung, dass die handelnden Personen öfter von Kindern als von Erwachsenen dargestellt werden (man denke etwa an das traditionsreiche Salzburger Adventsingen, das alljährlich ein soziologisch wie geographisch sehr gemischtes Publikum ins Große Festspielhaus lockt 
und bei dem man Lied- und Szenentexte hören kann, die manchen in der Literaturgeschichte zitierten sehr nahe stehen).

5 Natürlich eignet sich der Dialekt als authentisches Mittel zur sprachästhetischen Gestaltung alltagstypischer Situationen, fröhlicher Anlässe oder geläufiger Sozialpraktiken, aber er kann auch zum Vehikel sozialen Protests, ideologischer Subversion oder auch „karnevalesker“ Sinnlichkeit (cf. Ekellieder³) werden.

6 Für einen guten Teil des Korpus gilt, dass die Texte nicht von Vertretern jener Gesellschaftsschicht stammen, denen sie in den Mund gelegt werden, sondern von Autoren, die z. B. mit der Darstellung des einfältigen Bauern oder des unattraktiven Landlebens komische Effekte erzielen wollen. Andererseits darf aber auch nicht übersehen werden, dass die Aufführung bukolischer Szenen im Dialekt durch Angehörige des Hofes eine beliebte Unterhaltungsform darstellte (die zeigt, dass auch Aristokraten den Dialekt - mehr oder weniger souverän - beherrschten). Die im 18. Jahrhundert immer brisanter werdende, von sowohl konfessioneller als auch kulturprestigegesteuerter Rivalität geprägte Varietätenfrage kommt bei der Analyse der Texte in den unterschiedlichsten Konstellationen ins Spiel. Die Frage nach dem verbindlichen Schriftstandard wird zugunsten einer importierten Norm geregelt. Für den Gebrauchswert des Dialekts legt die Literaturgeschichte viele Zeugnisse vor, von denen jedes für sich sensibel interpretiert werden will. Aus den Texten lässt sich beispielsweise der Eindruck ableiten, dass vom weiblichen Geschlecht eine größere Nähe zum Standard erwartet wird (außer in pastourellenartigen Texten, in denen ein Aristokrat um die Gunst eines Landmädchens wirbt). tangiert das kulturelle Selbstverständnis des hier behandelten geographischen Raums schließlich ganz zentral in der Auseinandersetzung um Hanswurst und Kasperl; sie füllt folgerichtig auch das Schlusskapitel des Werks.

Die zahlreichen abgedruckten Beispieltexte sind nach einer Reihe von (auf S.607f offengelegten) Editionskriterien typographisch vereinheitlicht, in die Orthographie wird dagegen nur minimal eingegriffen (Auflösung schreibökonomischer Abkürzungen, keine Unterscheidung der s-Allographe zugunsten von s). Wörter und Konstruktionen, die nach Einschätzung der Autoren Verständnisprobleme hervorrufen könnten, werden unmittelbar im Anschluss an den Text übersetzt bzw. paraphrasiert oder erklärt (z.B. Pfoad] Hemd; Reinel] kleiner, flacher Topf; I wir eng bän ößn] Ich werde euch beim Essen; Geimayr] Wirtshaus in Wels, im Dezember 1781 Sammelpunkt der Protestanten zur Registrierung).

Das Buch ist sehr leserfreundlich geschrieben und sollte nicht nur Fachleute diverser Disziplinen, sondern auch interessierte Laien ansprechen. Lediglich im Einleitungsteil zum Kapitel Körper und Sinnlichkeit meint man ein gewisses Unbehagen der Autoren zu verspüren, das durch einen Wechsel in einen akademisch abgehobeneren Diskurs kompensiert wird. 51 Abbildungen unterschiedlichster Art - Titelblätter von Flugschriften, Noten-Autographen, Karikaturen etc. - illustrieren den Text. Das Literaturverzeichnis ist mit seinen etwa 500 Titeln sichtlich auf möglichst vollständige Erfassung des relevanten Schrifttums angelegt. Weit über eintausend Fußnoten sind Zeugnisse einer umfassenden Dokumentation und Aufarbeitung des Materials. Ein reich bestücktes Sach-, ein Orts- und ein Personenregister ${ }^{4}$ versprechen den Benützern auch Erfolg bei punktuellen Recherchen. Man wird dieser Literaturgeschichte den Rang eines Standardwerks zuerkennen dürfen. 


\section{NOTES}

1. Schreibende Frauen sind im Einzugsbereich der hier behandelten Literatur kaum je sichtbar in Erscheinung getreten; Therese von Artner (1772-1829) ist „die einzige namentlich bekannte

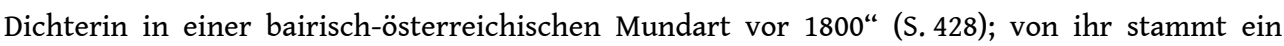
Glückwunschgedicht anlässlich der Hochzeit eines evangelischen Predigers.

2. Die Verfasser weisen an mehreren Stellen darauf hin, dass die Chance von Texten auf schriftliche Fixierung und Archivierung in Klöstern naturgemäß größer ist als in schriftfernen Milieus, wobei die Erwartung, dass sich im klerikalen Umfeld vorrangig sakral gefärbte Texte finden würden, nicht der Realität entspricht, weil auch weltliche Gedichte und Lieder „fixer Bestandteil der klösterlichen Unterhaltungskultur dieser Zeit" (S. 435) waren und profane Anlässe wie bestandene Prüfungen oder didaktische Bedürfnisse der Schulorden (cf. S. 441f.) als Auslöser für literarische Betätigung in Dialektform fungieren konnten.

3. Mit Norbert Elias ist man ja geneigt anzunehmen, dass allzu unappetitliche Liedtexte dem Vordringen der Schamgrenze seit dem 18. Jahrhundert zum Opfer gefallen wären. Die Autoren der Literaturgeschichte kommentieren das einschlägige Korpus in diesem Sinn: „Der heute zum Teil nicht mehr nachvollziehbare Reiz dieser grausigen Schock-Kunst kann verschieden gedeutet werden; manche werden ihr Normbewusstsein bestätigt gesehen oder dessen kurzzeitige Aufhebung genossen haben, anderen war es vielleicht Hilfe, eigene Phobien zu kontrollieren, für viele werden quasi-voyeuristische Motive zur schaurigen Unterhaltung beigetragen haben. In manchen Fällen aber wird es auch eine bewusste Provokation des Gegners gewesen sein“ (S. 495). Diese Art von Liedgut ist nach wie vor lebendig. Bei einer privaten Feier gab kürzlich ein Tiroler Berufsmusiker einschlägige Proben aus seinem Repertoire, die große Ähnlichkeit mit den Texten hatten, die in der Literaturgeschichte wiedergegeben sind.

4. Die Vollständigkeit des Personenregisters wurde nur stichprobenartig überprüft. Nicht aufgenommen sind Namen von Forschern, auch wenn sie im Fließtext und nicht nur in den Fußnoten erwähnt werden. Eine zufällig entdeckte Lücke: Der Name Bachtin erscheint mehrfach im Werk, er wird aber nur an einer Stelle (S. 295) registriert, nicht dagegen z.B. auf S. 474. 